\title{
Angiogenic factors: role in esophageal cancer, a brief review
}

\author{
Kátia Ladeira $^{1,2,3} \cdot$ Filipa Macedo $^{4} \cdot$ Adhemar Longatto-Filho $^{2,3,5,6,7} \cdot$ Sandra F. Martins ${ }^{2,3,8} \mathbb{C}$
}

Received: 6 July 2017 / Accepted: 11 December 2017 / Published online: 16 December 2017

(c) The Japan Esophageal Society and Springer Japan KK, part of Springer Nature 2017

\begin{abstract}
Esophageal cancer has an aggressive behavior with rapid tumor mass growth and frequently poor prognosis; it is known as one of the most fatal types of cancer worldwide. The identification of potential molecular markers that can predict the response to treatment and the prognosis of this cancer has been subject of a vast investigation in the recent years. Among several molecules, various angiogenic factors that are linked to the tumor development, growth, and invasion, such as VEGF, HGF, angiopoietin-2, IL-6, and TGF-B1, were investigated. In this paper, the authors sought to review the role of these angiogenic factors in prognosis and hypothesize how they can be used as a treatment target.
\end{abstract}

Keywords Esophageal cancer · HGF · Angiopoietin-2 · IL-6 · VEGF · TGF-B1

\section{Introduction}

Esophageal cancer has an aggressive tumor growth and poor prognosis and a 5-year overall survival rate between 15 and $25 \%$ [1]. It is one of the most fatal types of cancer worldwide. For esophageal squamous cell carcinoma (SCC), the overall 5-year survival rate is $\sim 20 \%$ [2]. The identification of potential molecular markers that can predict the response to treatment and the prognosis of the disease has been subject of a vast investigation in the last years.

Various studies indicated that factors that shape the tumor microenvironment might influence patient's response and

Sandra F. Martins

sandramartins@med.uminho.pt

1 Portuguese Oncology Institute, Lisbon, Portugal

2 Life and Health Sciences Research Institute (ICVS), School of Health Sciences, University of Minho, Campus de Gualtar, 4710-057 Braga, Portugal

3 ICVS/3B's-PT Government Associate Laboratory, Braga/Guimarães, Portugal

4 Portuguese Oncology Institute, Coimbra, Portugal

5 Molecular Oncology Research Center, Barretos, São Paulo, Brazil

6 Pathology Department, Hospital Braga, Braga, Portugal

7 Laboratory of Medical Investigation (LIM) 14, Faculty of Medicine, University of Sao Paulo, Sao Paulo, Brazil

8 Surgery Department, Coloproctology Unit, Hospital Braga, Braga, Portugal outcome. An inflammatory reaction promotes tumor proliferation, invasion, and angiogenesis [3]. Beyond a certain size, tumors are not further supported by diffusion, but undergo "an angiogenic switch" which supports further tumor growth and metastasis, with formation of new blood vessels within the tumor [4]. Based on that, various cytokines, including interleukin (IL)-6, IL-8 and tumor necrosis factor (TNF)- $\alpha$, are critical in the pathogenesis of numerous cancers. In particular, IL-6 serum levels have been reported to be elevated in patients with esophageal carcinoma [5], but its role in this cancer remains unclear. IL-6 plays a vital role in cell proliferation, migration, invasion, apoptosis, angiogenesis, growth, and differentiation of cancer cells [6]. Other angiogenic factors involved are vascular endothelial growth factor (VEGF), hepatocyte growth factor (HGF), and fibroblast growth factor (FGF) [7]. Several studies indicate that HGF is over-expressed in squamous cell carcinoma (SCC) tissue specimen and serum levels are associated with survival and clinicopathological parameters such as distant metastases [8]. Another angiogenic factor associated with poor survival is FGF [9]. Other studies have suggested an important role of the hypoxia-inducible factor-1 (HIF-1) as an inducer for angiogenic factors in hypoxic tumor microenvironment [10]. Among genes whose expression is regulated by HIF-1, VEGF is well documented as a key angiogenic factor [11]. Correlation of expression of these two factors with clinical outcome has been widely investigated in a variety of human cancers including esophageal cancer [12]. 
This paper reviews the role of angiogenic factors involved in development, growth, and proliferation of esophageal cancer, as well as their role in prognosis and, ultimately, how can they be used as a specific target for treatment.

\section{Materials and methods}

A PubMed research was realized focusing angiogenesis in esophageal cancer. The Mesh database with the terms: "Esophageal cancer/angiogenesis [mesh] and angiogenic factors/esophageal neoplasms [mesh]" was used.

A total of 30 articles were collected. Five articles were excluded from this review, because they studied the most suitable compartment for the assessment of the various angiogenic factors and did not study the molecule itself.

\section{Results and discussion}

\section{Hepatocyte growth factor}

HGF and its receptor c-Met play an important role in the development of various cancer types. It is a growth factor directed to epithelial cells that is important in embryogenesis and mediates defense to tissue damage in adults; HGF stimulation of vascular endothelial cells promotes migration, proliferation, protease production, invasion, and organization into capillary-like tubes. It binds c-Met (tyrosine kinase receptor) as exclusive ligand induces the activation of oncogenic pathways, angiogenesis, and scattering of cells, leading to metastasis [13].

The Met gene encodes the tyrosine kinase receptor for hepatocyte growth factor (HGF)/scatter factor (SF), and exposure of Met-expressing cells to this lead to proliferation, migration, invasion, and branching morphogenesis [14]. Numerous studies have shown that HGF/SF-Met is involved in esophageal SCC. Ren et al. [15] found that there was no significant correlation between serum HGF concentration and patients' gender, age, and tumor and node stage, in SCC. However, serum HGF levels were significantly higher in patients with $\mathrm{M} 1 \mathrm{a} / \mathrm{b}$ disease, and patients whose tumor was at stage III or IV also had higher HGF level compared with those who had stage I or II disease. The median survival of patients with low serum HGF levels $(n=75)$ was 34.7 months, and was significantly better than that of patients with high HGF levels $(n=74)$ at 15.8 months $(P=0.014)$; respective 2-year survival figures were 63.5 versus $37.0 \%$. The data also showed that HGF increased the secretion of other angiogenic factors, as VEGF and IL-8, in a dose-dependent manner. HGF-induced IL-8 and VEGF expression was found to be dependent on extracellular signal-regulated kinase (ERK) signaling pathways. The inhibition of ERK activation reduced HGF-induced IL-8 and VEGF expression, suggesting that this pathway is important in HGF-mediated production of angiogenic factors. Overexpression of c-Met was reported by Saeki et al. [16] in ESCC tumor cells and cell lines. These results provide a foundation for the hypothesis that HGF participates in angiogenesis via autocrine/paracrine mechanisms.

\section{Angiopoietin-2/follistatin}

Angiopoietin-2 is a critical regulator of tumor angiogenesis. Studies have shown a significant correlation of Angiopoietin-2 expression to tumor invasion and metastasis in various human cancers, but little is known about the serum Angiopoietin-2 levels in esophageal squamous cell cancer (ESCC) and its precursors. Zhou et al. investigated the role of Angiopoietin-2 in screening for ESCC and its precursors. The authors reported that serum Angiopoietin-2 levels were $22.0 \pm 5.5$ in normal group, $(21.3 \pm 3.2)$ in esophagitis, $20.5 \pm 3.3$ in hyperplasia, $24.0 \pm 5.0$ in early ESCC, and $29.8 \pm 5.0 \mathrm{U} / \mathrm{ml}$ in invasive ESCC group; it was significantly higher in the early ESCC than in hyperplasia group $(P=0.009)$, and the highest level was observed in invasive ESCC group $(P=0.000)$. The sensitivities of serum Angiopoietin-2 to early and invasive ESCC were 23.1 and $78.6 \%$, respectively. Therefore, the authors concluded that serum Angiopoietin-2 level is related to carcinogenesis and progression of ESCC, but it cannot be used to screen for early ESCC [17].

Dreikhausen et al. [18] reported a retrospective exploratory study to verify the association of angiogenic factors with prognosis in esophageal SCC and adenocarcinoma of the esophagogastric junction (AEG). In this study, the levels of most angiogenic factors in AEG and SCC were similar, but the differences of these two entities with respect to angiogenesis were indicated by the different expression of Angiopoietin-2 and Follistatin. They found significant higher median tissue levels of the two angiogenic factors in SCC patients as compared to AEG patients. Furthermore, they can be correlated with other angiogenic factors-high HGF and Follistatin expression in the tumor tissue were associated with poor patient's prognosis.

\section{IL-6}

IL-6 is one of the most dysregulated cytokines in cancer, acting to inhibit cell apoptosis and accelerating angiogenesis by the activation of KAK/STAT3 and PI3K/AKT signaling pathways [3]. STAT3 plays an important role in tumor progression, behavior, and epithelial-mesenchymal transition (EMT)-associated changes [19].

Zhao et al. [20] demonstrated that cancerous cells of esophageal SCC expressed significantly higher levels of IL-6 
when compared with normal epithelia $(P=0.0021)$. It was used an IL-6 silencing vector and it was observed that it significantly reduced the proliferation of the two cell lines used in this study compared with the control-vector-transfected cells $(P=0.0013)$ and, in addition, the silencing vector significantly attenuated the growth of tumors in the xenograft mouse models $(P=0.0031)$; furthermore, marked inhibition of tumor invasion was observed in the IL-6 silenced vector group in the two tumor models. It was hypothesized that IL-6 has a direct role or indirect role in angiogenesis and esophageal cancer progression. By inhibiting IL-6, tumor growth was found to be significantly inhibited in vitro and in vivo, and tumor growth and invasive ability was significantly attenuated $(P<0.05)$. In addition, the IL-6 silencing vector markedly reduced the presence of Ki-67 (an important proliferation marker) and microvessel density, indicating that down-regulation may affect tumor growth and inhibition; it also increased E-cadherin and matrix metalloproteinase (MMP)-9 expressions, and reduced the secretion of vascular endothelial growth factor (VEGF) and cluster of differentiation (CD)-31 (a nuclear protein), that mediated endothelial cell-cell interactions and actively participated in angiogenesis. These results indicated that EMT-associated changes and angiogenesis are interrelated and involved in aggressive tumor growth in esophageal cancer.

Many studies suggested that IL-6 provides a suitable in vivo environment for the growth and development of tumor cells, and reported that it may lead to resistance to certain anticancer drugs by promoting angiogenesis and cell mobility [21].

The hypothesis that IL- 6 may be an important trigger in the progression of angiogenesis and endothelial tube formation within the tumor is well described, and IL-6 may be a promising target for the treatment of esophageal cancer.

\section{VEGF/HIF-1}

HIF-1 is a heterodimeric basic helix-loop-helix protein composed of HIF- $1 \alpha$ and HIF- $1 \beta$ subunits [22]. Under normoxic conditions, the HIF-1 $\alpha$ subunit is subjected to ubiquitination and proteasomal degradation, whereas in hypoxia, its degradation is inhibited, and its expression increases rapidly [23]. Tumors derived from cells lacking HIF- $1 \alpha$ or HIF-1 $\beta$ show significantly reduced vascularization and often reduced growth rates compared with parental cells [24]. A key angiogenic factor whose gene expression is regulated by HIF-1 is the vascular endothelial growth factor (VEGF) [11]. VEGF is a family of the platelet-derived growth factor composed of 6 VEGF members including VEGF-A, VEGF-B, VEGF-C, VEGF-D, VEGF-E, and placenta growth factor (PIGF) [25]. Under hypoxic conditions within the tumor cells, HIF- $1 \alpha$ binds to a DNA recognition sequence in the promoter of target genes, including the VEGF gene [26]. Thus, under hypoxic conditions, VEGF transcription is up-regulated by HIF, and in addition, hypoxia promotes the stabilization of VEGF mRNA by proteins that bind to sequences in the $3^{\prime}$ untranslated region ( $3^{\prime}$ UTR) of the mRNA [27]. The prognostic significance of VEGF expression has been reported in gastric, colon, breast, bladder, and esophageal carcinomas, as well as in other malignancies [28]. The previous studies for gastrointestinal malignancies have focused on expression of VEGF-C in relation to lymph node metastasis and survival, including gastric, esophageal, and colorectal carcinomas [29]. Inhibition of HIF-1 $\alpha$ results in down-regulation of VEGF and increased apoptosis of cancer cells, indicating that HIF- $1 \alpha$ may serve as a potential target for cancer therapy while supporting its role as a key factor in tumor angiogenesis and tumor progression. HIF- $1 \alpha$ is absent in most normal tissues [30].

Recent studies have shown that HIF- $1 \alpha$ expression correlates with VEGF expression and high expression of these two factors is generally considered as poor prognostic predictors in various human cancers including esophageal cancer. Kimura et al. reported a 39\% of high expression of HIF- $1 \alpha$ in 32 out of their 82 ESCC tumor specimens and concluded that higher expression of HIF- $1 \alpha$ correlated positively with vascular invasion and microvessel density within the tumor but not tumor stage, individual TNM factors, or survival for the entire patient cohort [31]. In contrast, Tzao et al. showed a higher rate of high HIF-1 $\alpha$ expression, 52 in 85 (61.2\%), of resected tumor specimens; the expression of HIF- $1 \alpha$ correlated positively with tumor stage and inversely with patients' survival. They speculate that preoperative induction therapy may alter HIF-1 $\alpha$ expression in ESCC [32].

The previous studies rarely investigated relationship between expression of HIF- $1 \alpha$ and VEGF-D in the same series. Expression of HIF- $1 \alpha$ is associated with that of VEGF-C in ESCC and both of these two factors predict patient's survival [33]. Tzao et al. have also demonstrated that expression of VEGF-D, relative to VEGF-C, was also associated with expression of HIF- $1 \alpha$ in resected ESCC, for which supported by a significant concordance (69.5\%) between expression of HIF- $1 \alpha$ and VEGF-D. Taken together, these results may imply that different types of VEGF may function synergistically with HIF- $1 \alpha$ in promoting tumor angiogenesis. However, author reveals that this speculation needs further investigation on their gene expression and biological functions in relation to tumor angiogenesis using in vitro and/or in vivo models [34].

The identification of selective HIF-1 inhibitors would not only be useful for the potential therapeutic implications but also for their application as analytic tools to further define the role of HIF in human cancers. Continued efforts have been made in the development of HIF inhibitors, such as synthetic polyamides, that inhibit HIF-1 DNA binding [35], 
and more recently, it was identified small molecule inhibitors (e.g., 103D5R) that selectively blocks HIF-1 protein synthesis [34]. On the other hand, bevacizumab, an antiVEGF monoclonal antibody, has been proven to be an useful target therapy option in a variety of cancers [35], and ramucirumab (a VEGFR-2 inhibitor that prevents VEGF binding in endothelial cells) is actually recommended by the National Comprehensive Cancer Network (NCCN) as a second-line therapy for metastatic esophageal adenocarcinoma [36].

\section{TGF- $\beta 1$}

Cytokines of the transforming growth factor (TGF)- $\beta$ superfamily are multifunctional proteins that regulate a variety of biological processes, including cell growth, differentiation, apoptosis, and morphogenesis [37], inhibiting epithelial cell proliferation. Loss of the growth-inhibitory response to TGF- $\beta$ is found in many tumor cells [38] and the excess TGF- $\beta$ that results to this sensibility loss may act on tumor cells and stromal cells to facilitate invasion and metastasis [39]. In tumors, it acts early in carcinogenesis as a tumor suppressor but acts later as a stimulator of tumor invasion by prompting extracellular matrix production and angiogenesis, stimulating tumor proliferation, and inhibiting host immune functions. Loss or resistance of this inhibitory effect to TGF- $\beta$ has been reported in many tumor cells and elevated systemic TGF- $\beta 1$ levels have been reported in various cancers [40-45], as well as increased local TGF- $\beta 1$ has been observed in many cancer cells [47]. Local expression of TGF- $\beta 1$ was related to tumor progression in esophageal cancer, in contrast to systemic TGF- $\beta 1$ level [43]. It is known that plasma TGF- $\beta 1$ level may be influenced by systemic inflammation or a chronic disease, and TGF- $\beta 1$ may be secreted from normal tissues as well as tumors.

In a study, plasma TGF- $\beta 1$ level was obtained from venous blood of the azygos vein instead of a peripheral vein and was measured and correlated with pathological findings and survival of patients with esophageal cancer. The azygos vein is responsible for venous return from the esophagus, and his level of plasma TGF- $\beta 1$ may reflect tumor progression and condition more directly and accurately than the TGF- $\beta 1$ level in other veins [47]. Plasma for normal controls could not be obtained from the azygos vein of healthy volunteers, so it was used the mean value measured from peripheral veins [43]. A significant correlation between the TGF- $\beta 1$ level measured from the azygos vein and metastasis to distant lymph nodes in esophageal cancer $(P=0.0396)$ was observed, suggesting that elevated TGF- $\beta 1$ exhibits the effects of angiogenesis, extracellular matrix production, and immune suppression, contributing to tumor development and metastasis to distant lymph nodes [48]. It was observed that the survival rates of patients with high TGF- $\beta 1$ levels (levels above the mean value of healthy controls) were significantly lower than those of patients with low TGF- $\beta 1$ levels $(P=0.0317)$ and the TGF- $\beta 1$ level measured from the azygos vein was an independent prognostic factor of overall survival $(P=0.0474)$. According to these results, the TGF$\beta 1$ level in the azygos vein may reflect tumor condition and viability more appropriately than local expression does. High TGF- $\beta 1$ levels predict poor prognosis in patients who would eventually have cancer recurrence in lymph nodes or other organs. The authors concluded that the level of plasma TGF- $\beta 1$ measured from the azygos vein is a useful prognostic marker in patients with esophageal cancer, but further studies with more patients and follow-up those for longer periods will be needed.

\section{Fibroblast growth factor}

The expression of basic fibroblast growth factor (b-FGF) in squamous cell carcinoma of the esophagus was immunohistochemically investigated in tissue specimens from 79 patients with squamous cell carcinoma of the esophagus who underwent curative esophagectomy without preoperative chemotherapy or radiation therapy. Tumor cells that expressed b-FGF were found in 41 patients (51.9\%). The mean vascular density of b-FGF-positive tumors $\left(47.9 / \mathrm{mm}^{2}\right)$ was significantly lower than that of b-FGF-negative tumors $\left(67.2 / \mathrm{mm}^{2}\right)(P=0.014)$, and the survival rate of patients with b-FGF-positive tumors was significantly higher than those with b-FGF-negative tumors $(P=0.033)$. The authors concluded that the expression of b-FGF may be associated with promotion of angiogenesis and a good candidate as a prognostic factor in squamous cell carcinoma of the esophagus [49] (Fig. 1).

\section{Conclusion}

Angiogenesis is essential for tumor growth and its spread, including invasion and metastasis. However, rather than being controlled by means of a simple mechanism, the control of tumor angiogenesis may be mediated by several angiogenic factors. HGF, VEGF, HIF-1, IL-6, FGF, TGF-B1, angiopoietin-2, and follistatin are some of these factors, with several studies demonstrating their importance in esophageal cancer, mainly in squamous cell carcinoma of the esophagus. The best studied angiogenic factor is VEGF, with several studies in the literature. It also seems to be the most important in the prognosis of patients with esophageal cancer. In the REGARD trial, it was recently shown that treatment with an antibody against VEGF receptor 2 (VEGFR-2) in monotherapy, ramucirumab, can prolong survival in patients with advanced gastric and esophageal cancer. However, it is also known that other angiogenic factors have an important 
Fig. 1 Angiogenic factors previously described and their role in esophageal cancer angiogenesis

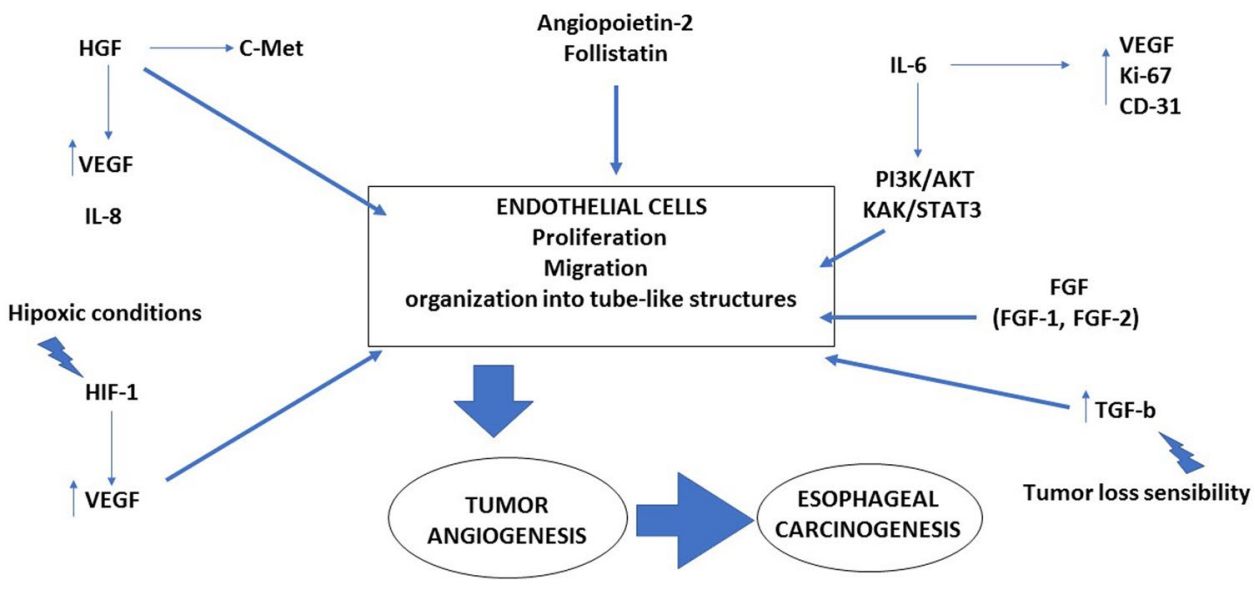

role in cancer development and progression, like IL-6, HGF, angiopoietin-2, and TGF-B1. These can be used as prognostic markers but, more importantly, as potential therapeutic targets. However, it is consensual that more studies and trials are needed.

\section{Compliance with ethical standards}

Ethical statement This article does not contain any studies with human or animal subjects performed by any author(s).

Conflict of interest The authors have no conflicts of interest and received no financial support for this study.

\section{References}

1. Pennathur A, Gibson MK, Jobe BA, et al. Oesophageal carcinoma. Lancet. 2013;381:400-12.

2. Jemal A, Siegel R, Ward E, et al. Cancer statistics 2009. CA Cancer Clin. 2009;59:225-49.

3. Coussens LM, Werb Z. Inflammation and cancer. Nature. 2002;420:860-7.

4. Hanahan D, Weinberg RA. Hallmarks of cancer: the next generation. Cell. 2011;144:646-74.

5. Guo Y, Xu F, Lu T, et al. Interleukin-6 signaling pathway in targeted therapy for cancer. Cancer Treat Rev. 2012;38:904-10.

6. Kishimoto T. Interleukin-6: from basic science to medicine 40 years in immunology. Annu Rev Immunol. 2005;23:1-21.

7. Oshima Y, Yajima S, Yamazaki K, et al. Angiogenesis-related factors are molecular targets for diagnosis and treatment of patients with esophageal carcinoma. Ann Thorac Cardiovasc Surg. 2010;16:389-93.

8. Xu Z, Wang $\mathrm{S}, \mathrm{Wu} \mathrm{M}$, et al. TGF beta1 and HGF protein secretion by esophageal squamous epithelial cells and stromal fibroblasts in oesophageal carcinogenesis. Oncol Lett. 2013;6:401-6.

9. Sugiura K, Ozawa S, Kitagawa Y, et al. Co-expression of aFGF and FGFR-1 is predictive of a poor prognosis in patients with esophageal cancer. World J Gastroenterol. 2005;11:2188-92.

10. Folkman J. How is blood vessel growth regulated in normal and neoplastic tissue? Cancer Res. 1986;11:467-73.

11. Leo C, Giaccia AJ, Denko NC. The hypoxic tumor microenvironment and gene expression. Semin Radiat Oncol. 2004;14:207-14.
12. Kimura S, Kitadai Y, Tanaka S, et al. Expression of hypoxia inducible factor (HIF)- $1 \alpha$ is associated with vascular endothelial growth factor expression and tumour angiogenesis in human oesophageal squamous cell carcinoma. Eur J Cancer. 2004;40:1904-12.

13. Birchmeier C, Birchmeier W, Gherardi E, et al. Metastasis, motility and more. Nat Rev Mol Cell Biol. 2003;4:915-25.

14. Bottaro DP, Rubin JS, Faletto DL, et al. Identification of the hepatocyte growth factor receptor as the c-met proto-oncogene product. Science. 1991;251:802-4.

15. Ren Yi, Cao Brian, Law Simon, et al. Hepatocyte growth factor promotes cancer cell migration and angiogenic factors expression: a prognostic marker of human esophageal squamous cell carcinomas. Clin Cancer Res. 2005;3:6190-7.

16. Saeki H, Oda S, Kawaguchi H, et al. Concurrent overexpression of Ets-1 and c-Met correlates with a phenotype of high cellular motility in human esophageal cancer. Int J Cancer. 2002;98:8-13.

17. Zhou YZ, Fang XQ, Li H, et al. Role of serum angiopoietin-2 level in screening for esophageal squamous cell cancer and its precursors. Chin Med J (Engl). 2007;120:1216-9.

18. Dreikhausen L, Blank S, Sisic L, et al. Association of angiogenic factors with prognosis in esophageal cancer. BMC Cancer. 2015;15:121-7.

19. Bromberg J. Stat proteins and oncogenesis. J Clin Invest. 2002;109:1139-42.

20. Zhao Z-F, Li J-X, Ye Rui, et al. Interleukin-6 as a potential molecular target in esophageal squamous cell carcinoma. Oncol Lett. 2016;11:925-32.

21. Schindler C, Levy DE, Decker T. JAK-STAT signaling, from interferons to cytokines. J Biol Chem. 2007;282:20059-63.

22. Michiels C, Minet E, Mottet D, et al. Regulation of gene expression by oxygen: NF-kappaB and HIF-1, two extremes. Free Radic Biol Med. 2002;1:1231-42.

23. Wang GL, Jiang BH, Rue EA, et al. Hypoxia inducible factor 1 is a basic helix-loop-helix-PAS heterodimer regulated by cellular $\mathrm{O}_{2}$ tension. Proc Natl Acad Sci USA. 1995;1:5510-4.

24. Carmeliet P, Dor Y, Herbert JM, et al. Role of HIF-1 $\alpha$ in hypoxiamediated apoptosis, cell proliferation and tumor angiogenesis. Nature. 1998;394:485-90.

25. Veikkola T, Alitalo K. VEGFs, receptors and angiogenesis. Semin Cancer Biol. 1999;9:211-20.

26. Pugh CW, Ratcliffe PJ. Regulation of angiogenesis by hypoxia: role of the HIF system. Nat Med. 2003;9:677-84.

27. Dibbens JA, Miller DL, Damert A, et al. Hypoxic regulation of vascular endothelial growth factor mRNA stability requires the cooperation of multiple RNA elements. Mol Biol Cell. 1999;10:907-19. 
28. Ellis LM, Liu W, Fan F, et al. Role of angiogenesis inhibitors in cancer treatment. Oncology (Williston Park). 2001;15:39-46.

29. Amioka T, Kitadai Y, Tanaka S, et al. Vascular endothelial growth factor- $\mathrm{C}$ expression predicts lymph node metastasis of human gastric carcinoma invading the submucosa. Eur J Cancer. 2002;15:1413-9.

30. Zhong H, De Marzo AM, Laughner E, et al. Over-expression of hypoxia-inducible factor $1 \alpha$ in common human cancers and their metastases. Cancer Res. 1999;59:5830-5.

31. Aebersold DM, Burri P, Beer KT, et al. Expression of hypoxiainducible factor-1 alpha: a novel predictive and prognostic parameter in the radiotherapy of oropharyngeal cancer. Cancer Res. 2001;12:2911-6.

32. Tzao C, Lee S-C, Tung H-J, et al. Expression of hypoxia-inducible factor (HIF)-1alpha and vascular endothelial growth factor (VEGF)-D as outcome predictors in resected esophageal squamous cell carcinoma. Dis Markers. 2008;25:141-8.

33. Olenyuk BZ, Zhang GJ, Klco JM, et al. Inhibition of vascular endothelial growth factor with a sequence-specific hypoxia response element antagonist. Proc Natl Acad Sci USA. 2004;101:16768-73.

34. Tan C, de Noronha RG, Roecker AJ, et al. Identification of a novel small-molecule inhibitor of the hypoxia-inducible factor 1 pathway. Cancer Res. 2005;65:605-12.

35. Sandler A. Bevacizumab in non-small cell lung cancer. Clin Cancer Res. 2007;355:4613-6.

36. Samson P, Lockhart AC. Biologic therapy in esophageal and gastric malignancies: current therapies and future directions. J Gastrointest Oncol. 2017;8(3):418-29.

37. Massagué J. TGF- $\beta$ signal transduction. Annu Rev Biochem. 1998;67:753-91.

38. Lahm $\mathrm{H}$, Odartchenko $\mathrm{N}$. Role of transforming growth factor $\beta$ in colorectal cancer. Growth Factors. 1993;9:1-9.

39. S.-W I, Volpert OV, Bouck NP, et al. Smad4/DPC4-mediated tumor suppression through suppression of angiogenesis. Proc Natl Acad Sci USA. 2000;7:9624-9.

40. Shirai $Y$, Kawata $S$, Tamura $S$, et al. Plasma transforming growth factor- $\beta 1$ in patients with hepatocellular carcinoma comparison with chronic liver diseases. Cancer (Phila). 1994;73:2275-9.
41. Junker U, Knoefel B, Nuske K, et al. Transforming growth factor- $\beta$ 1 is significantly elevated in plasma of patients suffering from renal cell carcinoma. Cytokine. 1996;8:794-8.

42. Tsushima H, Kawata $S$, Tamura $S$, et al. High levels of transforming growth factor- $\beta 1$ in patients with colorectal cancer: association with disease progression. Gastroenterology. 1996; 110:375-82.

43. Saito H, Tsujitani S, Oka S, et al. The expression of transforming growth factor- $\beta 1$ is significantly correlated with the expression of vascular endothelial growth factor and poor prognosis of patients with advanced gastric carcinoma. Cancer (Phila). 1999;86:1455-62.

44. Shariat SF, Kim JH, Andrews B, et al. Preoperative plasma levels of transforming growth factor- $\beta 1$ strongly predict clinical outcome in patients with bladder carcinoma. Cancer (Phila). 2001;92:2985-92.

45. Narai S, Watanabe M, Hasegawa $H$, et al. Significance of transforming growth factor- $\beta 1$ as a new tumor marker for colorectal cancer. Int J Cancer. 2002;97:508-11.

46. Natsugoe S, Xiangming C, Matsumoto M, et al. Smad4 and transforming growth factor- $\beta 1$ expression in patients with squamous cell carcinoma of the esophagus. Clin Cancer Res. 2002;8:1838-42.

47. Chevallier JM, Vitte E, Derosier C, et al. The thoracic esophagus: sectional anatomy and radiosurgical applications. Surg Radiol Anat. 1991;13:313-21.

48. Fukuchi M, Miyazaki T, Fukai Y, et al. Plasma level of transforming growth factor- $\beta 1$ measured from the azygos vein predicts prognosis in patients with esophageal cancer. Clin Cancer Res. 2004;10:2738-41.

49. Nakamura T, Ozawa S, Kitagawa Y, et al. Expression of basic fibroblast growth factor is associated with a good outcome in patients with squamous cell carcinoma of the esophagus. Oncol Rep. 2005;14:617-23. 